\title{
28 Research Square \\ Development and Patient Experience Evaluation of a Gentle Atomizer for Nasal Drug Delivery
}

Uzzam Ahmed Khawaja ( $\square$ uzzamahmedkhawaja@gmail.com )

Jinnah Medical and Dental College https://orcid.org/0000-0002-8442-5174

Syed A. A. Rizvi

Hampton University School of Pharmacy

Boliang Gong

Bona Broadcasting Ltd

Waiman Yeung

Bona Broadcasting Ltd

Marcos A. Sanchez-Gonzalez

Lake Erie College of Osteopathic Medicine

Gustavo Ferrer

Aventura Hospital and Medical Center

\section{Research Article}

Keywords: atomizer, drug delivery, nasal spray, fluid dynamics. open-angle swirling

Posted Date: April 13th, 2021

DOI: https://doi.org/10.21203/rs.3.rs-381105/v1

License: (c) (i) This work is licensed under a Creative Commons Attribution 4.0 International License.

Read Full License 


\section{Abstract}

Background: Recently, nasal drug delivery has emerged as a convenient modality for the treatment of multiple, allergic, infectious, and inflammatory sinonasal conditions. Some patients report discomfort and irritation associated with the pressure and flow generated by using typical atomizers which may lead to decrease patient compliance and lower satisfaction. We sought to develop a rather gentle atomizer for nasal irrigation that would ameliorate the discomfort associated with nasal drug delivery. Herein, we report the development of an atomizer via fluid dynamics modeling and evaluating the patient experience.

Methods: Using computational flow dynamics modeling, we assessed the distribution of atomized droplets to compare the conventional narrow-angle (NA) Vs a new open-angle swirling effect (OSE) atomizer. The goal for the new atomizer was to generate a swirling effect by opening a spray cone from a tapered nozzle bottle at different head tilted positions to determine the most efficient one that would enhance drug delivery and aid in patient comfort. Once the best computational model was generated and the atomizer developed, a group of 13 healthy volunteers consented to participate in the performance evaluation of the two atomizers (NA and OSE). Participants gauged both atomizers for ergonomics, pressure, and comfort for drug delivery (saline). The participants' feedback was collected using the nozzle tip sensory attributes questionnaire.

Results: Atomizer Fluid Dynamics: The comparison was made between NA and OSE using the latest recommendation on the intranasal delivery angle of $45^{\circ}$ with the nozzle $1.5 \mathrm{~cm}$ into the nose bypassing the nasal valve. The NA went $7 \mathrm{~cm}$ into the nose, and hence generated high frictional pressure in the nasal mucosa. On the other hand, the OSE deliverd doses between $3-7 \mathrm{~cm}$, thus generating less pressure. Patient Experience: $60 \%$ of the participants encountered irritation with the NA atomizer such as pain and a burning sensation. About $20 \%$ felt the OSE to be soft and pleasant, while $90 \%$ reported a misting sensation.

Conclusion: The results of the present study suggest that OSE is a viable and more comfortable system to deliver intranasal therapies. These findings shed light on our understanding of the fluid flow dynamics to design extra efficient and comfortable atomizers to maximize drug delivery in the nasal cavity and potentially increase patient satisfaction as well as compliance.

\section{Introduction}

The nasal route is of great significance for efficient delivery of therapeutic agents due to rich vascular supply resulting in a swift systemic and local response, particularly when rapid absorption and effect are required such as in the treatment of pain, erectile dysfunction, migraine, seizures, insomnia, panic attacks, rigidity in parkinsonism, hot flashes, emesis, and cardiovascular events [1, 2]. For instance, some of the commonly used nasal products commercially available are Zomig ${ }^{\circledR}$ (zolmitriptan), Imitrex ${ }^{\circledR}$ (sumatriptan), and Stadol NS (butorphanol tartrate) for the treatment of migraine; Aerodiol ${ }^{\circledR}$ (estradiol 
hemihydrate) for menopausal syndrome treatment; and PecFent ${ }^{\circledR}$, and Instanyl ${ }^{\circledR}$ (fentanyl) for management of severe pain [3]. Interestingly, when compared with injections or pills for the delivery of drugs with the ability to bypass the blood-brain barrier, nasal sprays are rendered more efficient [4]. The nasal route of drug delivery also has preeminence over other administration routes like oral or intravenous, in particular, non-invasiveness, self-administration, lesser time to effect onset, and greater bioavailability because of hepatic 1st pass metabolism shunning [5]. Despite the stated advantages, intranasal drug delivery encounters several challenges and limitations for the absorption of therapeutic agents with low intrinsic permeability, hydrophilic molecules such as peptides, proteins, and nucleotides as well as due to the intrinsic characteristics of the nasal cavity such as rapid mucociliary clearance, and enzymatic degradation [6, 7]. Taken together, the aforementioned factors may have a negative impact on the patients' perceptions and use experience ultimately leading to poor compliance and unoptimzed drug delivery.

Patient compliance towards a prescribed treatment regimen plays a pivotal role in disease management and therapeutic success. Hence, patient acceptance of, and satisfaction with, the prescribed therapeutic regimen is paramount. Recent studies demonstrate that patients' attitudes towards a therapeutic regimen are influenced not only by the agent's sensory triggering characteristics but also by the ease and solace of administration [8-10]. Cardinal factors affecting patient adherence comprise variations in delivery devices, operating maneuvers, and usage feasibility. Since an appealing association has been noted between patient predilection and compliance, its vital to regard patient acceptability when prescribing medications, particularly those to be taken intranasally $[11,8,12,13]$. Variability and obscurity present in instructions demonstrate a lack of knowledge on the most acceptable methods to administer a nasal spray device $[14,15]$. Kundoor and Dalby (2011), reported the area of deposition decreased with increased viscosity, but this was mediated by an increase in droplet size and a narrowing of the spray plume [19]. If all the aforementioned factors are well-thought-out while developing an intranasal drug delivery device, a better patient experience followed by improved patient compliance is highly likely.

Accordingly, the primary goal of the present study was to develop an effective and comfortable nasal spray unit or intranasal delivery system to improve patient experience and potentially compliance. Consequently, the main objective of the present study was twofold (i) to develop a vortex-generating swirling atomizer, and (ii) to evaluate patient satisfaction and comfort using the device. We hypothesized that a circular, open-angle swirling effect (OSE) atomizer pattern with droplets size greater than $5 \mathrm{mc}$ will deliver an adequate dose at the center of the nasal cavity with the least pressure-related side effects and improved patient experience.

\section{Methods}

Using computational flow dynamics modeling, we assessed the distribution of atomized droplets to compare and contrast the conventional narrow-angle (NA) vs a new open-angle swirling effect atomizer. The goal was to generate a swirling effect by opening a spray cone from a tapered nozzle bottle at different head tilted positions to determine the most efficient one that would enhance drug delivery and 
aid in patient compliance. Briefly, the design of a rotary atomization nasal drug delivery system was mainly aimed at solving the disadvantages of the existing nasal drug delivery device, such as causing nasal discomfort to patients and cannot ensure the liquid reaches the accurate drug delivery position, and hence failing to achieve the desired efficacy. According to the nasal aerodynamics principle, the rotary atomizing nasal cavity is designed as a special structure in the injector nozzle [20]. It is atomized into a rotating column before the liquid spray, and a hollow wide-angle cone shape is formed after the liquid spray is discharged. According to the characteristics of the medicine and the requirement of medicine, the size of the spray particle is fixed, the spray shape and the spray angle are determined to ensure that the sprayed medicine is not made to the mucous membrane. It can also enhance the intranasal transmission of spray particles to achieve effective drug distribution.

Once the best computational model was generated a group of 13 healthy volunteers consented to participate in this series of tests in writing (Tables $1 \& 2$ ). Participants blindly evaluated a commonly used atomizer NA vs open-angle OSE for delivery (saline), ergonomics, pressure, and comfort. Both atomizer models were introduced $17 \mathrm{~mm}$ into the nose at an angle of $45^{\circ}$, based on the latest recommendations by Saikat Basul et al. [21]. Briefly, Saikat Basul et al. recommendations indicate that the atomizer should be inserted five-eighths of an inch into the nostril and be held at a $35^{\circ}-45^{\circ}$ angle for a better more uniform distribution of the fluid. The feedback of the participants was noted on a nozzle tip sensory attributes questionnaire and clear-cut instructions were given in the "How to Use a Nasal Spray Properly" illustration [21].

Table 1

Data are presented as $\mathrm{M} \pm \mathrm{SEM}$. Patients characteristics in the cohort with respect to sex and medical history.

\begin{tabular}{|c|c|c|c|}
\hline \multicolumn{4}{|c|}{ Subjects Characteristics $(N=13)$} \\
\hline \multirow[t]{2}{*}{ Sex } & Male & 4 & $33.1 \%$ \\
\hline & Female & 9 & $66.9 \%$ \\
\hline \multirow[t]{5}{*}{ Past Medical History } & Chronic Rhinitis & 2 & $20.4 \%$ \\
\hline & Intermittent Rhinitis & 3 & $21.0 \%$ \\
\hline & Asthma & 3 & $23.3 \%$ \\
\hline & Sinusitis & 1 & $8.0 \%$ \\
\hline & none & 4 & $27.3 \%$ \\
\hline Age & $39.23 \pm 3.86$ & & \\
\hline
\end{tabular}


Table 2

Participants' individual responses of the atomizer tip sensory attributes questionnaire. Note: Conventional narrow-angle (NA) vs a new open-angle swirling effect (OSE).

\begin{tabular}{|lllll|}
\hline $\begin{array}{l}\text { Which you fee is a } \\
\text { better designed tip? }\end{array}$ & $\begin{array}{l}\text { Most soft \& gentle } \\
\text { spray delivery tip? }\end{array}$ & $\begin{array}{l}\text { Tip with } \\
\text { greater } \\
\text { pressure? }\end{array}$ & $\begin{array}{l}\text { Most } \\
\text { soothing } \\
\text { tip }\end{array}$ & $\begin{array}{l}\text { Irritation } \\
\text { encountered? } \\
\text { (NA) }\end{array}$ \\
\hline OSE & OSE & NA & OSE & YES \\
\hline OSE & OSE & NA & OSE & NO \\
\hline OSE & OSE & NA & OSE & YES \\
\hline OSE & OSE & NA & OSE & NO \\
\hline OSE & OSE & NA & OSE & NO \\
\hline OSE & OSE & NA & OSE & YES \\
\hline OSE & OSE & NA & OSE & YES \\
\hline OSE & NA & OSE & YES \\
\hline OSE & OSE & NA & OSE & YES \\
\hline OSE & OSE & NA & OSE & YES \\
\hline OSE & OSE & NA & OSE & NO \\
\hline OSE & OSE & NA & OSE & NO \\
\hline OSE & OSE & NA & OSE & YES \\
\hline
\end{tabular}

\section{Results}

\section{Fluid Dynamics Modeling}

The narrow-angle atomizer went $7 \mathrm{~cm}$ into the nose, and hence generated high frictional pressure in the nasal mucosa. On the other hand, the open-angle swirling effect atomizer reached maximum delivery doses between $3-7 \mathrm{~cm}$, thus generating less pressure against the mucosa.

\section{Droplet size distribution, Spray pattern, and Plume Geometry}

The results of particle size distribution comparison between NA and OSE are presented in Tables A and B in the supplementary info-1. The results were reported for D10, D50, and D90 which are the volume diameters of the particles at $10 \%, 50 \%$, and $90 \%$ cumulative volume respectively, and the span value (spread of particles). Of note, the soft nozzle OSE was found to decrease particle size distribution (D10, D50, and D90) as well as the span. The spray pattern and plume geometry results are presented in Tables $C$ and D in supplementary info 2 . The terms, SP $30 \mathrm{~mm}$ denoted spray pattern from while PG $30 \mathrm{~mm}$ is plume geometry from $30 \mathrm{~mm}$ distance inside the nose. The ovality ratio of typical aqueous nasal spray 
has a typical range of 1.1 to 1.2 , in our case, the ovality ratios were higher i.e., 1.295 and 1.268 for NA and OSE, respectively.

\section{Patient Experience}

A total of 13 patients participated in this study (Table 1). After using the nasal spray their responses were recorded onto a nozzle tip sensory attributes questionnaire. The distance of $3 \mathrm{~cm}$ from the nozzle tip was chosen as the point to measure the peak of the total dose delivery during the spray pattern test. If the average user measures $12 \mathrm{~cm}, 6 \mathrm{~cm}$ is the center of the cavity. The nozzle is introduced $1.8 \mathrm{~cm}$ plus $4 \mathrm{~cm}$ of spray performance peak guarantee an adequate dose without pressure damage to the mucosa. All the participants reported that OSE had a better design, more gentle delivery, more soothing, but with less pressure than the NA. A total of $60 \%$ of participants reported that the NA was irritant with pain as the most commonly reported experience whereas the OSE was found to feel like a mist by the participants (Table 2).

\section{Discussion}

We sought to develop a gentler atomizer for nasal irrigation that would ameliorate the discomfort associated with drug delivery. The novel OSE atomizer reached maximum delivery doses between 3-7 $\mathrm{cm}$, thus generating less pressure against the mucosa which was perceived as gentler and more comfortable by the participants. The results suggest that the OSE atomizers may be an alternative userfriendly strategy that has the potential to improve the patient experience of topical delivery inside the human nose with enhanced compliance implications.

The nasal passage is about $12 \mathrm{~cm}$ long and about $5 \mathrm{~cm}$ high. The nasal cavity is much narrower above where the olfactory nerve is located. For optimal drug delivery recent research concluded that the atomizer tips need to be introduced $1.8 \mathrm{~cm}$ into the nostril independently of the spray pattern angle. In agreement with our hypothesis, a circular, slightly OSE-generated spray pattern with droplets size greater than $5 \mathrm{~cm}$ will be delivered an adequate dose at the center of the nasal cavity with the least pressurerelated side effects.

Clinical studies by Tay SY et al. (2012), demonstrate gentle inspiration technique to enhance the intranasal spread of sprayed particles in patients suffering from allergic rhinitis, however, the airway geometry was not a part of this study [22]. Furthermore, multiple nasal spray devices make use of a pressure-swirl atomizer [23] which implies some swirl component within the initial particle velocities. Limitations of nose-to-brain delivery exist through the use of this method, and consist of a relatively small volume for the administration of the drugs, limited surface area of the olfactory epithelium, a short period of retention for the absorption of the drug, and elicit a nasal secretion upon delivery of the drug [24].

The nose is a suitable site for the administration of various drugs and vaccines, however, the ultimate potential has not been understood yet as the structural limitations concerning the nasal anatomy, physiology, and aerodynamics might curb the potential and need further exploration. Pivotal 
enhancements in the technical device industry have illustrated noticeable reliable in vitro results; thereby leading to improved clinical performance. Testing of in vitro performance is undoubted of great essence to evaluate the quality of the product, the predictive value for evaluating the in vivo clinical performance remains contentious. CFD simulations of nasal aerodynamics may play a crucial role in the initial stages of device designing; followed by future advances leading to improved predictive value. Human in vivo deposition and clearance studies are imperative in delivering valuable data particularly if current development warranting wide quantification and tissue attenuation correction is applied [25-27]. Controlled clinical trials are needed to evaluate alterations in both the symptoms and the functional framework, and additionally to establish the efficacy of novel drugs/devices [28].

Both spray pattern and plume geometry are important parameters for evaluating the performance of a nasal pump. Various factors can affect the spray pattern and plume geometry, including the size and shape of the nozzle, the design of the pump, and the characteristics of the formulation. The spray pattern

is characterized by the $D_{\max }$ (longest diameter), $D_{\min }$ (shortest diameter), and Ovality Ratio $\left(D_{\max } / D_{\min }\right)$. Plume geometry is characterized by the spray angle and plume width. The overall average ovality results in Table 4 ( $A$ and $B$ ) confirm that the two methods produce similarly shaped patterns. The plume geometry (cone angle) measurements show a modest increase in the angle and width of the spray with OSE. Guo et al. (2005) observed that sprays with wider plume angles had increased posterior deposition. Meanwhile, the computational simulations of Kimbell et al. (2007) failed to show any effect of plume angle on the deposition.

\section{Conclusion}

In sum, the present study demonstrated the development and patient experience of a novel atomizer for intranasal use. The OSE enhanced nasal drug delivery system introduces a new approach to overcome the structural limitations of the current conventional nasal delivery due to the dynamics of the nasal valve which may ultimately translate into clinical benefit for the patients. The novel OSE atomizer nasal delivery device is suitable for many drugs including medicines for the treatment of allergic rhinitis, influenza, and it may even be of great use and value in the treatment of COVID-19. Since the patient experience was enhanced, we believe this could lead to improved medication compliance and therapeutic effectiveness. Clinical studies to test the effectiveness and compliance of patients including the successful administration of intranasal therapies using the OSE are warranted.

\section{Declarations}

\section{Funding:}

Not applicable

\section{Conflicts of interest/Competing interests:}

Not applicable 
Availability of data and material:

The datasets generated during and/or analysed during the current study are available from the corresponding author on reasonable request.

\section{Declarations of interest:}

none

\section{References}

1. Djupesland, P.G. Nasal drug delivery devices: characteristics and performance in a clinical perspective-a review. Drug Deliv. and Transl. Res. 3, 42-62 (2013). https://doi.org/10.1007/s13346012-0108-9

2. T.Y. Ting, I. Gonda, E.M. Gipps, Microparticles of polyvinyl-alcohol for nasal delivery 0.1. generation by spray-drying and spray-desolvation, Pharm. Res. 9 (10) (1992) 1330-1335.

3. Illum L. Nasal drug delivery-recent developments and future prospects. J Control Release. 2012;161:254-63.

4. Bleske BE, Warren EW, Rice TL, Shea MJ, Amidon G, Knight P. Comparison of intravenous and intranasal administration of epinephrine during CPR in a canine model. Ann Emerg Med. 1992;21(9):1125-1130. doi:10.1016/s0196-0644(05)80657-2

5. Dufes C, Olivier JC, Gaillard F, Gaillard A, Couet W, Muller JM. Brain delivery of vasoactive intestinal peptide (VIP) following nasal administration to rats. Int J Pharm. 2003;255(1-2):87-97. doi:10.1016/s0378-5173(03)00039-5

6. Lim ST, Forbes B, Brown MB, Martin GP. Physiological factors affecting nasal drug delivery. In: Touitou E, Barry BW, editors. Enhancement in drug delivery. Boca Raton: CRC Press; 2007.

7. Pillon DJ, Arnold JJ, Meezan E. Nasal delivery of peptides. In: Touitou E, Barry BW, editors. Enhancement in drug delivery. Boca Raton: CRC Press; 2007.

8. Bachert C, El-Akkad T: Patient preferences and sensory comparisons of three intranasal corticosteroids for the treatment of allergic rhinitis. Ann Allergy Asthma Immunol. 2002, 89: 292-297. 10.1016/S1081-1206(10)61957-6.

9. Storms WW: Introduction: patient preference of inhaled nasal corticosteroids. Allergy Asthma Proc. 2001, 22 (Suppl 1): S1-S3.

10. Blaiss MS: Efficacy, safety, and patient preference of inhaled nasal corticosteroids: a review of pertinent published data. Allergy Asthma Proc. 2001, 22 (Suppl 1): S5-S10.

11. Meltzer EO, Bardelas J, Goldsobel A, Kaiser H: A preference evaluation study comparing the sensory attributes of mometasone furoate and fluticasone propionate nasal sprays by patients with allergic rhinitis. Treat Respir Med. 2005, 4: 289-296. 10.2165/00151829-200504040-00007. 
12. Stokes M, Amorosi SL, Thompson D, Dupclay L, Garcia J, Georges G: Evaluation of patients' preferences for triamcinolone acetonide aqueous, fluticasone propionate, and mometasone furoate nasal sprays in patients with allergic rhinitis. Otolaryngol Head Neck Surg. 2004, 131: 225-231. 10.1016/j.otohns.2004.04.011.

13. Khanna P, Shah A: Assessment of sensory perceptions and patient preference for intranasal corticosteroid sprays in allergic rhinitis. Am J Rhinol. 2005, 19: 316-321.

14. Benninger, M. S. et al. Techniques of intranasal steroid use. Otolaryngology-Head and Neck Surgery 130(1), 5-24 (2004)

15. Kundoor, V. \& Dalby, R. N. Effect of formulation-and administration-related variables on deposition pattern of nasal spray pumps evaluated using a nasal cast. Pharmaceutical Research 28(8), 18951904 (2011).

16. D.O. Frank, J.S. Kimbell, D. Cannon, S.S. Pawar, J.S. Rhee, Deviated nasal septum hinders intranasal sprays: a computer simulation study, Rhinology 50 (3) (2012) 311-318.

17. D.O. Frank, J.S. Kimbell, S. Pawar, J.S. Rhee, Effects of anatomy and particle size on nasal sprays and nebulizers, Otolaryngol.-Head Neck Surg. 146 (2) (2012) 313-319.

18. P.G. Djupesland, Nasal drug delivery devices: characteristics and performance in a clinical perspective - a review, Drug Deliv. Transl. Res. 3 (1) (2013) 42-62.

19. V. Kundoor, R.N. Dalby, Effect of formulation- and administration-related variables on deposition pattern of nasal spray pumps evaluated using a nasal cast, Pharm. Res. 28 (8) (2011) 1895-1904.

20. Gungor, A. A. (2013). "The aerodynamics of the sinonasal interface: the nose takes wing-a paradigm shift for our time." Int Forum Allergy Rhinol 3(4): 299-306.

21. Basu, S., Holbrook, L.T., Kudlaty, K. et al. Numerical evaluation of spray position for improved nasal drug delivery. Sci Rep 10, 10568 (2020). https://doi.org/10.1038/s41598-020-66716-0

22. Tay SY, Chao SS, Mark KTT, Wang DY. Comparison of the distribution of intranasal steroid spray using different application techniques. International Forum of Allergy \& Rhinology. 2012; 6(11):1204-1210. https://doi.org/10.1002/alr.21807

23. Fung MC, Inthavong K, Yang W, Tu J. CFD Modeling of Spray Atomization for a Nasal Spray Device. Aerosol Science and Technology. 2012; 46(11):1219-1226.

https://doi.org/10.1080/02786826.2012.704098

24. H. Wu, K. Hu, X. JiangFrom nose to brain: understanding transport capacity and transport rate of drugs Expert Opin. Drug Deliv., 5 (2008), pp. 1159-1168, 10.1517/17425247.5.10.1159

25. Djupesland PG, Skretting A. Nasal deposition and clearance in man: comparison of a bidirectional powder device and a traditional liquid spray pump. J Aerosol Med Pulm. 2012. doi:10.1089/jamp.2011.0924

26. Skretting A, Djupesland PG. A new method for scintigraphic quantification of deposition and clearance in anatomical regions of the human nose. Nucl Med Comm. 2009;30(8):629-38

27. Darquenne C. Aerosol deposition in health and disease. J Aerosol Med Pulm. 2012;25(3):140-7 
28. Laube B. Devices for aerosol delivery to treat sinusitis. J Aerosol Med. 2007;20(Suppl):5-18.

\section{Supplementary Files}

This is a list of supplementary files associated with this preprint. Click to download.

- GraphicalAbstract.jpg

- Dropletsizedistribustioncomparereport.docx

- SpraypatternPlumegeometrycomparereport.docx 